\title{
Vertrauensprobleme, Vertrauensformen und Vertrauensforschung
}

\author{
Versuch eines Überblicks
}

Simon Peng-Keller

Wer versucht, das Feld der Vertrauensforschung abzuschreiten, muss sich auf einen weiten Weg einstellen. ${ }^{1}$ Noch vor zwei Jahrzehnten ist dieses Forschungsgebiet leicht überblickbar gewesen. Seitdem ist es nicht nur stark gewachsen, sondern es hat sich in einzelne Forschungszweige ausgefächert, die nur sehr lose miteinander verbunden sind. Umso mehr drängt sich eine Orts- und Grenzabschreitung auf, die nicht nur auf die Unselbstverständlichkeit des Vertrauens reflektiert, sondern auch die Eigentümlichkeit des Nachdenkens über das Vertrauen. Dass das Vertrauen in derVielfalt seiner Formen (erst) in den letzten beiden Jahrzehnten des 20. Jahrhunderts zu einem beliebten Thema der Wissenschaften wurde, ist ein Faktum, das selbst erklärungsbedürftig ist. Nach Antony Giddens ist Vertrauen «in fundamentaler Weise mit den Institutionen der Moderne verbunden». ${ }^{2}$ Wie bereits Georg Simmel zu Beginn des letzten Jahrhunderts deutet Giddens den Prozess der Modernisierung und Globalisierung als eine Transformation von lokal begrenzten und eingebundenen Vertrauensbeziehungen $\mathrm{zu}$ weiter ausgreifenden Vertrauensnetzen. Das Anschwellen der Vertrauensforschung steht in Antwort auf diese Entwicklung. Dabei verflechten sich die empirische Vertrauensforschung und politische Vertrauens(krisen)diskurse in eigentümlicher Weise. Der folgende Versuch eines orientierenden Grenzgangs möchte auch solche Verflechtungen in den Blick nehmen. Sie beginnen bei den Wissenschaften selbst, deren Vertrauensbedarf unersättlich ist.

\section{Wissenschaftliches Vertrauen - Vertrauen in die Wissenschaft}

Wissenschaften leben vom Kredit, denen man ihnen zuteilt. Da es sich dabei um ein rares Gut handelt, geht das nicht ohne Ver-

1 Für hilfreiche Kommentare zu einer ersten Fassung dieses Beitrags danke ich I.U. Dalferth und A. Hunziker.

2 A. Giddens, Konsequenzen der Moderne. Frankfurt a.M. 1996, 39. 
teilkämpfe und gegenseitige Verdächtigungen ab. Besonders die harten sciences, die Tatsachenwissenschaften, treten hier gerne mit viel Selbstvertrauen auf. Sie geniessen nicht zuletzt deshalb grosses Vertrauen, weil sie mit dem Anspruch verbunden sind, in ihren Verfahren nicht auf Vertrauen, sondern auf Misstrauen und Kontrolle zu setzen. ${ }^{3}$ Obwohl auch in solchen Zusammenhängen längst nicht alles kontrolliert und nachgerechnet werden kann, und auch eine Naturwissenschaftlerin nicht ohne das Vertrauen in ihre Kolleginnen auskommt, scheint es Nicht-Experten im Allgemeinen einfacher zu fallen, der messenden und rechnenden Beobachtervernunft Kredit einzuräumen als etwa der historischen Vernunft, die es mit mehr oder weniger vertrauenswürdigen Quellen und mit stets problematisierbaren Glaubwürdigkeitseinschätzungen zu tun hat - und deshalb vielleicht selber in Sachen Vertrauenswürdigkeit schwerer einzuschätzen ist. Das robuste Selbstvertrauen der Naturwissenschaften zeugt von einem grossen Methodenvertrauen - einer Transformationsform des Vernunftvertrauens, das die abendländische Denkgeschichte lange begleitet hat, inzwischen aber im Zeichen «schwachen Vernunft von philosophischer Seite her in Frage steht. Der methodische Zweifel, den die Philosophie kultiviert, kann sich eben auch gegen ihr eigenes Vernunftvertrauen richten.

Die natural sciences können die Angemessenheit ihres Methodenvertrauens - anders als die Philosophie, die in ihren Reflexionswelten den Schatten des Fraglichen nicht abzustreifen vermag - durch handfeste Erfolge belegen. Bei aller Unschärfe vieler Ergebnisse sind die gewonnenen Einsichten doch hinreichend genau, um in technologischer Hinsicht innovativ zu wirken. Der Erfolg des naturwissenschaftlichen Methodenvertrauens verleitet $\mathrm{zu}$ problematischen Generalisierungen und zu einem überzogenen Misstrauen in die Erkenntniskraft der hermeneutischen Wissenschaften, die nicht messen und rechnen, sondern - in methodisch kontrollierter Weise entziffern, auslegen und interpretieren. Die Geschichtswissenschaften und die Theologie bilden im Kreise der hermeneutischen Disziplinen insofern eine besondere Gruppe, als sie in je unterschiedlicher Weise nicht (ganz) ohne den Rekurs auf Zeugenaussagen auskommen. Wissenschaftsfähig ist ein solcher Rekurs auf nicht kontrollierbare Aussagen dadurch, dass nicht nur die Glaubwürdigkeit der Zeugen, sondern auch ihre Täuschbarkeit kritisch geprüft werden.

\footnotetext{
3 Vgl. M. Gutmann/B. Rathgeber/T. Syed, Warum vertrauen wir auf Wissenschaften? Überlegungen zu Kriterien erzwungener Verlässlichkeit, in: M. Maring (Hg.), Vertrauen - zwischen sozialem Kitt und der Senkung von Transaktionskosten, Karlsruhe 2010, 45-70.
} 
Das Vertrauen in Experten und Expertensysteme, das nach Giddens ein Kennzeichen der (Spät-)Moderne ist, findet im Vertrauen in die Wissenschaften seinen paradigmatischen Fall. Dass es, wie alles Vertrauen, in die Irre gehen und hypertroph werden kann, ist kaum $\mathrm{zu}$ bestreiten. Strittig ist hingegen die Frage, ob die Leistungskraft beobachtender und instrumenteller Vernunft gemeinhin überschätzt wird und ob ein Übermass an dieser Form von Expertenvertrauen zu einer technologischen Kolonialisierung der Lebenswelt bzw. zu einer Verwissenschaftlichung der Alltagskultur geführt hat. Soll man den Wissenschafts- und Technologiekritikern, die solches behaupten, Vertrauen schenken? Oder zumindest der eigenen Wahrnehmung, dass die Welt, in der man sich zurechtzufinden hat, durch technologische Innovationen nicht nur einfacher, sondern auch komplexer, undurchschaubarer und manchmal auch gefährlicher wird?

\section{Vertrauen in Experten}

Das Vertrauen in die Wissenschaften stellt einen besonderen Fall des Expertenvertrauens dar, das sich als emergentes Phänomen mit zunehmender gesellschaftlicher Diversifizierung einstellt. Die vertrauenssichernde Funktion von sich selbst kontrollierenden Expertensystemen hat ihre reichlich bekannten Schattenseiten. Tendieren sie doch dazu, ihre eigene Vertrauenswürdigkeit $\mathrm{zu}$ überzeichnen und auch dort noch Vertrauen zu suggerieren, wo Misstrauen angesagt wäre. In den letzten Jahrzehnten entwickelten sich deshalb ergänzend zu den etablierten Expertensystemen der unterschiedlichen Professionen und bisherigen Versicherungsagenturen neue Formen von Kredit vergebenden Institutionen wie Kundenschutzorganisationen, Akkreditierungsstellen und Qualitätssicherungsinstitute, die ihre Hauptaufgabe darin finden, bereits bestehende Expertensysteme zu überprüfen und zu überwachen. Diese sekundären Expertensysteme profitieren nicht allein von einem Vertrauensvorschuss, den die älteren Systeme im Laufe einer pannenreichen Geschichte längst eingebüsst haben. Als Institutionen professionellen Misstrauens sind sie von vornherein auf der sichereren Seite. Beim Aufdecken der Fehler der Anderen können einem weniger Fehler passieren, als wenn man sich selbst etwa auf dem riskanten Feld der Medizin oder der Flug- und Atomtechnologie bewegt. Selbstkontrollorgane machen diesbezüglich allerdings eine Ausnahme: Spätestens wenn ein Flugzeug abstürzt oder ein Reaktor explodiert, werden auch die Kontrolleure kontrolliert. 
Die weiter wachsende und sich vervielfältigende Abhängigkeit von Expertensystemen führt auch zu ethischen Problemen. Sie steht in einem spannungsreichen Verhältnis zum breit abgestützten Ideal, sein Leben selbstbestimmt und authentisch $\mathrm{zu}$ führen. Zumindest die Kunst, den Experten in angemessener Weise zu misstrauen, lässt sich nicht nochmals an Experten delegieren. Wer dem geschulten Blick der Fachleute in jedem Fall mehr vertraut als seinen eigenen Sinnen, verlernt das Wahrnehmen und Urteilen. Innerhalb der Expertenwelten wiederholt sich das Problem: Sollen Fachleute, z.B. erfahrene Ärzte, im Zweifelsfall nicht eher ihrer Intuition vertrauen als ihren unbestechlichen, aber in bestimmter Hinsicht eben bornierten Messinstrumenten? Ellen J. Langer, Professorin für Psychologie in Harvard und damit Angehörige eines der weltweit renommiertesten Expertensysteme, kritisiert das blindeVertrauen in die diagnostischen und kurativen Fähigkeiten moderner Medizin. Ärztlichen Diagnosen und Behandlungsvorschlägen werde, so meint sie, gemeinhin zu unbefragt vertraut. Ist aber eine Kultivierung des Misstrauens in diesem Zusammenhang tatsächlich wünschenswert? Belegen nicht unzählige Studien zum Placebo-Effekt, dass die Heilung zu einem beträchtlichen Teil vom Vertrauen in ein bestimmtes Verfahren einerseits und in die behandelnde Person andererseits abhängt? Hat deshalb ein übervorsichtiger und misstrauischer Patient nicht geringere Heilungschancen als ein vertrauensseliger? Nur dann, wenn er sich in kompetenten Händen befindet, denen er sich anvertrauen könnte. Gegenüber von Scharlatanen fährt der Misstrauische besser. Komplexe Situationen erfordern eine ausgewogene Mischung von Vertrauen und Misstrauen. Grundsätzlich dürfte gelten, dass dort, wo viel $\mathrm{zu}$ verlieren ist, eine misstrauische Voreinstellung angebracht sein dürfte. ${ }^{4}$ Giddens These, dass Vertrauen in fundamentaler Weise mit den Institutionen der Moderne verbunden sei, ist insofern zu modifizieren, als mit der Frage nach dem angemessenen Vertrauen immer auch diejenige nach dem angemessenen Misstrauen auf dem Plan ist. Das zeigt sich insbesondere mit der modernen Institution par excellence: der parlamentarischen Demokratie.

\section{Vertrauen politisch}

Viele politische Vertrauensprobleme lassen sich unter die eben betrachtete Kategorie subsumieren. Sind doch auch Politikerinnen

\footnotetext{
4 K. Jones, Trust in Science and in Scientists, in: I. Shapiro u.a. (Hg.), Integrity and Conscience, New York 1998, 139-153, hier: 147.
} 
und Politiker Experten, die in vielerlei Hinsicht selber auf Experten angewiesen sind. Zudem spielen, stärker noch als in den meisten anderen Professionen, Versprechen eine zentrale und komplizierte Rolle: Neben den artikulierten Wahlversprechen und allfäligen Amtseiden bauen Politikerinnen und Politiker ihre Karriere auf dem Versprechen auf, den Aufgaben, die man ihnen überträgt, gewachsen zu sein, sie im Sinne ihrer Partei wahrzunehmen und die ihnen übertragene Macht nicht zu missbrauchen. Auch wo mit politischer Konkurrenz und kritischer medialer Aufmerksamkeit zu rechnen ist, liegt eine doppelte Versuchung nahe: einerseits zu viel zu versprechen und andererseits gewisse Versprechen, mit denen man sich finanzielle und anderweitige Unterstützung sichert, zu verheimlichen.

Entsprechend $\mathrm{zu}$ den tatsächlich geschehenen oder auch nur naheliegenden Vertrauensbrüchen wird Politikern - auch in Ländern wie der Schweiz und Deutschland - in Umfragen regelmässig eine viel weniger hohe Vertrauenswürdigkeit zugeschrieben als Feuerwehrleuten, Ärztinnen oder Pfarrern. Kann man sich damit beruhigen, dass eine Demokratie nicht allein auf solchen Zuschreibungen aufbaut, sondern sie auch das Misstrauen durch Gewaltenteilung, Meinungsfreiheit und ausgeklügelte Wahlprozesse institutionalisiert hat und so das Vertrauen in Politiker und politische Parteien durch Sanktionsmechanismen absichert? Muss man aber dann nicht wiederum den Kontrolleuren und den Kontrollmechanismen vertrauen können?

Misstrauen allein macht keinen Staat. Eine Demokratie lebt davon, dass ihre Bürgerinnen und Bürger sich mit ihrer politischen Lebensform und ihrenVertreter/innen identifizieren können und sich für sie engagieren. Um funktionieren zu können, bedarf ein demokratisches Gemeinwesen aber nicht nur ein gewisses Mass an Vertrauen in ihre Repräsentanten und des Vertrauens in die Mechanismen einer Demokratie, sondern dichte, sich überschneidende und gegenseitig stützende Vertrauens- und Solidaritätsnetzwerke:Verbände, Parteien, Gewerkschaften, gemeinnützige Organisationen, religiöse Gemeinschaften etc. Nach Shmuel N. Eisenstadt ist für die erfolgreiche Generalisierung und Stabilisierung von Vertrauen ein kontinuierlicher «Fluss des Vertrauens zwischen den Gesellschaftssektoren und den verschiedenen Zentren (...) entscheidend.» ${ }^{5}$

Eng verbunden mit dem Vertrauen in Politiker/innen und politische Systeme sind eine Vielfalt von ökonomisch geprägten

5 S. N. Eisenstadt,Vertrauen, kollektive Identität und Demokratie, in: M. Hartmann/ C. Offe (Hg.),Vertrauen. Die Grundlage des sozialen Zusammenhalts, Frankfurt a.M. 2001, 333-363, hier: 357. 
Vertrauensformen. Die empirische Vertrauensforschung hat sich ihnen in den letzten Jahren mit besonderer Intensität zugewandt.

\section{Ökonomische Vertrauensformen}

Die Verflechtung von Vertrauensdiskursen und Vertrauensforschung zeigt sich mit besonderer Deutlichkeit in der Ökonomisierung des Vertrauensvokabulars. Vertrauen wird als Investition eingeschätzt und als rares Gut gehandelt, das deshalb teuer ist. Es ist, so heisst es, eine unter globalisierten Bedingungen gefährdete Ressource. Doch sind diese Metaphern angemessen? Verbirgt eine solche Vertrauensrhetorik nicht den nicht ökonomisierbaren Charakter des Vertrauens? Man kann zumindest zu bedenken geben, dass sich Vertrauen durch den Gebrauch vermehrt und nicht aufzehrt wie knappe Ressourcen. ${ }^{6}$ Ein ökonomisch-sparsamer Umgang mit dem Vertrauen gefährdet diese Ressource mitunter stärker als ihre Verschwendung. Unbestreitbar ist jedenfalls, dass die Zuschreibung von Vertrauenswürdigkeit - der Inbegriff einer guten Reputation - als soziales Kapital betrachtet werden kann, das man leicht verspielen kann. Die spezifisch ökonomische Wertschätzung des Vertrauen lässt sich mit Martin Fiedler auf eine einfache Formel bringen: «Vertrauen ist gut. Kontrolle ist teuer.» ${ }^{7}$ Nicht vertrauen zu können, ist ebenso kostspielig wie leichtfertiges Vertrauen. Generalisiertes Misstrauen verhindert ökonomisch lukrative Kooperation, Vertrauen ermöglicht sie. Dabei laufen auch hier personales Vertrauen und Systemvertrauen ineinander:Weder das Vertrauen in die jeweiligen Akteure, mit denen man es konkret zu tun hat, noch das Vertrauen in die Kontroll- und Regulationsmechanismen des jeweiligen Marktes stehen für sich, sondern bedingen sich gegenseitig. Wie sich der globalisierte Markt durch zunehmende Verflechtung regionaler Märkte, Rechts- und Währungssysteme entwickelt hat, lässt sich historisch genau nachzeichnen. ${ }^{8}$ Dabei ist eine paradoxe Dynamik zu beobachten: Die zunehmende Vereinheitlichung der globalen Währungs- und Handelsmärkte vereinfacht nicht nur die

6 D. Gambetta, Können wir dem Vertrauen vertrauen?, in: M. Hartmann/C. Offe (Hg.), Vertrauen. Die Grundlage des sozialen Zusammenhalts, Frankfurt a.M. 2001, 204-237, hier: 221 und 236.

7 Zit. in H. Berghoff, Die Zähmung des entfesselten Prometheus? Die Generierung von Vertrauenskapital und die Konstruktion des Marktes im Industrialisierungs- und Globalisierungsprozeß des 19. Jahrhunderts, in: H. Berghoff/J. Vogel (Hg.), Wirtschaftsgeschichte als Kulturgeschichte. Dimensionen eines Perspektivenwechsels, Frankfurt a.M. 2004, 143-168, hier: 144.

${ }^{8}$ Vgl. den oben zitieren Tagungsband von Berghoff/Vogel. 
weltumspannenden ökonomischen Inter- und Transaktionen. Sie führt auch zu einer Steigerung von Komplexität und Interdependenz, die durch die bisherigen Instrumente des Risikomanagements kaum mehr überschauen sind. Reinhard Bachmann und Akbar Zaheer sehen in ihrer Einleitung zum 2006 erschienenen Handbook of Trust ${ }^{9}$ gerade darin den Hauptgrund für die gegenwärtige Konjunktur der Vertrauensforschung: Die fortschreitende Globalisierung erfordert in ihrer unüberschaubaren Eigendynamik nicht nur mehr Flexibilität, sondern auch mehr Vertrauen in Geschäftsbeziehungen. Denn mit grösserer Komplexität und Ungewissheit wird auch die Unvollständigkeit von Verträgen ständig grösser. Vertrauen transformiert diese nicht zu vermeidende Ungewissheit in ein akzeptiertes Risiko.

Der Sachverhalt, dass bei ökonomischen Vertrauensbeziehungen ausdrücklich oder implizit Risikokalkulationen im Spiel sind, wird in der Vertrauensforschung unterschiedlich aufgenommen. Nach James Coleman ${ }^{10}$ lässt sich Vertrauen als eine kalkülbasierte Entscheidung unter Risiko fassen, die sich formalisieren lässt. Es handle sich um eine Form von Kreditgewährung, die dadurch zu charakterisieren sei, dass die möglichen Verluste für den Vertrauensgeber grösser sind als der Gewinn, den man sich erhofft. Eine solche Berechenbarkeit von Vertrauen wird von Oliver Williamson, ${ }^{11}$ Martin Endreß, ${ }^{12}$ Peter Eberl ${ }^{13}$ und anderen mit guten Gründen kritisiert. Zum einen blendet Colemans Formalisierung einen entscheidenden Punkt der Vertrauensgabe aus, nämlich den lebensweltlichen Entstehungszusammenhang von Vertrauen im Allgemeinen und den Einfluss des Vertrauensgebers auf die Vertrauensbereitschaft des Empfängers im Besondern. ${ }^{14}$ Die Konzentration auf eine individualistische Entscheidungslogik verzeichnet den sozialen Charakter

\footnotetext{
9 R. Bachmann/A. Zaheer (Hg.), Handbook of Trust, Cheltenham UK/Northampton MA 2006

${ }^{10}$ J. S. Coleman, Grundlagen der Sozialtheorie, Bd. 1: Handlungen und Handlungssysteme, München 1991, 126.

11 O.Williamson, Calculativeness, trust and economic organization, in: Journal of Law and Economics 36 (1993), 453-386.

12 M. Endreß,Vertrauen, Bielefeld 2002, 37ff.

${ }_{13}$ P. Eberl,Vertrauen und Management. Studien zu einer theoretischen Fundierung des Vertrauenskonstruktes in der Managementlehre. Stuttgart 2003, 69ff.

${ }^{14}$ Vgl. M. Endreß, Vertrauen und Vertrautheit - Phänomenologisch-anthropologische Grundlegung, in: M. Hartmann/C. Offe (Hg.), Vertrauen. Die Grundlage des sozialen Zusammenhalts, Frankfurt a.M./New York 2001, 161-203, hier: 185: «Vertrauen kann (...) nicht angemessen als eine einseitige Beziehung bzw. als seinseitige Vorleistung gedeutet werden. Vielmehr verweist der Vertrauensbegriff auf ein wechselseitiges Verhältnis:Vertrauen ist ein Interaktionsprodukt und keine prinzipiell vorgängig zu erbringende Leistung.»
} 
der Vertrauensgenese und Vertrauensstabilisierung. Problematisch an Colemans Konzeptionalisierungsversuch ist zum anderen die auch für die spieltheoretische Vertrauensforschung typische Gleichsetzung von trust und entrusting, also von Vertrauen mit einem beobacht- und messbaren Ressourcentransfer. ${ }^{15}$ Schliesslich kann man Lars Herzbergs bereits 1988 geäusserte Kritik, dass in der neueren Vertrauensdiskussion Vertrauen (trust) oft mit Sich-Verlassen (reliance) verwechselt werde, auch auf Colemans Konzept anwenden. ${ }^{16}$

Es sind mindestens drei Fälle denkbar, in denen einer Person im Rahmen der von Coleman genannten Bedingungen eine bestimmte Geldsumme anvertraut wird, ohne dass dies ein Fall von Vertrauen darstellt. Wenn ich aufgrund von wirksamen Sanktionsmechanismen sicher sein kann, dass der andere nicht anders kann, als mir den Betrag zurückzugeben, braucht es meinerseits kein Vertrauen, ihm mein Geld anzuvertrauen. Ebensowenig handelt es sich um einen Akt von Vertrauen, wenn ich aus moralischen oder sozialen Gründen gezwungen bin, ihm den Betrag anzuvertrauen. Und schliesslich gibt es das reine Risikoverhalten, das von einerVertrauensgabe manchmal kaum unterscheiden lässt. Da es sich bei vielen Vertrauensexperimenten um Spielanlagen handelt, die zu Kalkül und Risiko einladen, ist besonders der letzte Fall von einigem Gewicht:Wie lässt sich gewährleisten, dass bei dem sogenannten Trust-Game tatsächlich Vertrauen und nicht einfach Spiellust oder Risikofreude gemessen wird? Dass Probanden im Risk-Game mit einem Zufallsgenerator deutlich andere Formen des Einsatzes entwickeln als anonym bleibende Mitspieler im Trust-Game, und sich diese Differenz durch neurowissenschaftliche Befunde erhärten lässt, bedeutet einen ersten Schritt zu einer experimentellen Eingrenzung des Vertrauensphänomens. ${ }^{17} \mathrm{Ob}$ damit das Phänomen hinreichend erfasst ist, muss weiter untersucht und diskutiert werden. Es fragt sich insbesondere, ob auch innerhalb des kooperativen Verhaltens, das im Trust-Game untersucht wird und gegen ein Risikoverhalten gegenüber einem Zufallsgenerator abgegrenzt

\footnotetext{
15 So das Urteil von M. Levi, The Transformation of a Skeptic: What Nonexperimentalists Can Learn from Experimentalists, in: E. Ostrom/J. Walker (Hg.), Trust and Reciprocity. Interdisciplinary lessons from experimental research, New York 2003, 373-380, hier: "[M] uch oft he experimental research oft he past conflates trust an cooperation or trust and reciprocity.» Vgl. auch D. M. Rousseau et al., Not so different after all: A cross-discipline view of trust, in: Academy of Management Review 23 (1998), 393-404, hier 395: «Trust is not a behavior (e.g., cooperation), or a choice (e.g., taking a risk), but an underlying psychological condition that can cause or result from such actions.»

${ }^{16}$ L. Herzberg, On the attitude of trust, in: Inquiry, 31 (1988), 307-322, hier: $311 f$.

${ }^{17} \mathrm{Vgl}$. den Beitrag von J. Engelmann in diesem Band.
} 
werden kann, experimentell zwischen einer vertrauensbestimmten Form von Kooperation und einem Risikoverhalten unterschieden werden kann, das auch auf die Ressourcen von Empathie und geschenktem Vertrauen in eigennütziger Weise zurückgreift. Lässt sich experimentell unterscheiden zwischen (1.) einer Kreditvergabe aus reiner Spiellust, (2.) einer berechnenden Kreditvergabe, die auf die Vertrauenswürdigkeit des anderen spekuliert, und (3.) einer Kreditvergabe, in der sich ein nicht instrumentelles Vertrauen manifestiert, das bereit wäre, um der vertrauensvollen Kooperation willen auf Gewinnmaximierung zu verzichten?

Die Generierung und Stabilisierung vonVertrauen in Handels- und Kreditbeziehungen ist das vielleicht gewichtigste, aber bei weitem nicht das einzige Feld ökonomisch orientierterVertrauensforschung. Auch in der Betriebswirtschafts- und Managmentlehre avancierte das Vertrauen in den letzten Jahren zu einem wichtigen, wenn auch umstrittenen Thema. Die zunehmende Globalisierung und der beschleunigte Wandel des Arbeitsmarktes führten auch in diesen Bereichen zu eine Fülle von neuen Herausforderungen, die kaum ohne Vertrauen zu lösen sind. Dabei werfen insbesondere das Verhältnis zwischen Vertrauen und Kontrolle sowie die (Un-) Verfügbarkeit von ersterem komplizierte Fragen auf. Nach Peter Eberl können «Vertrauensbeziehungen (...) nicht institutionalisiert werden. Sie sind Ausfluss informeller Prozesse in Organisationen», die allerdings in gewissen Graden indirekt beeinflusst werden können. ${ }^{18}$ Die gegenwärtige Managmentlehre tendiert dazu, Vertrauen nicht als Substitut, sondern als Supplement zu angepassten Kontrollmechanismen $\mathrm{zu}$ betrachten, die einen sicheren Rahmen für inner- und interorganisationale Vertrauenskommunikation bilden.

\section{Ethos des Vertrauens}

Wenn Vertrauen (trust) und mit Risiken verbundener Ressourcentransfer (entrusting) zu unterscheiden sind, und Vertrauen gegenüber von Zutrauen dem anderen nicht nur eine spezifische Kompetenz zuschreibt, sondern auch davon ausgeht, dass er oder sie das geschenkte Vertrauen nicht missbraucht, dann ist mit Vertrauen unweigerlich auch die Ethik im Spiel. Wer jemandem Vertrauen schenkt, erwartet nicht nur, dass dieses Vertrauen nicht ausgenützt wird, sondern geht davon aus, dass Vertrauen nicht ausgenützt wer-

${ }^{18}$ P. Eberl, Art. Vertrauen, in: G. Schreyögg/A. v. Werder (Hg.), Handwörterbuch Unternehmensführung und Organisation, Stuttgart ${ }^{4} 2004$, 1596-1604, hier: 1603. 
den darf. ${ }^{19}$ Das ist auch für eine ökonomische Betrachtungsweise bedeutsam, mag es auch die Problemstellung verkomplizieren. Zum einen fragt sich, ob es unter einem ethischen Gesichtspunkt vertretbar ist, Vertrauensbeziehungen aus rein ökonomischem Eigeninteresse aufzubauen und $\mathrm{zu}$ pflegen. Auch wenn man davon ausgeht, dass in ökonomischen Beziehungen alle Beteiligten wissen, dass die Vertrauenskommunikation eine ökonomischen Zielsetzung verfolgt, stellt sich die Frage nach den Grenzen einer solchen Instrumentalisierung. Peter M. Blau vertrat bereits 1964 die These, dass das Vertrauen, das die Ökonomie so dringend braucht, nicht ökonomisch herzustellen ist: "Only social exchange tends to engender feelings of personal obligation, gratitude, and trust; purly economic exchange as such does not.» ${ }^{20}$ Wenn die Ökonomie von externen Ressourcen lebt, so kann sie der Frage nach dem Verhältnis zwischen ökonomischem Kalkül und moralischer Verpflichtung nicht ausweichen: Beinhaltet Vertrauen nicht die Bereitschaft, das geschenkte Vertrauen auch dann nicht zu enttäuschen, wenn es sich ökonomisch gesehen nicht mehr auszahlt?

Zum anderen stellt uns die ökonomische Verflechtung von Kalkül und Vertrauen vor die Frage, was es an sozialen und politischen Voraussetzungen braucht, damit eine solch vertrauensbasierte Ökonomie überhaupt funktioniert. Offenkundig leben ökonomische Vertrauensbeziehungen von einer Vertrauenskultur, die sie selbst nicht nur nicht garantieren können, sondern die sie sogar durch ihre Instrumentalisierung des Vertrauens selbst zu zerstören drohen. Auf die kulturelle Einbettung des merkantilen Vertrauens hatte bereits Max Weber hingewiesen. In seiner Wirtschaftsethik der Weltreligionen beschreibt Weber die christlich-abendländische Entwicklung als Universalisierung eines ethisch begründeten Vertrauens.

Es sei «von sehr erheblicher ökonomischer Bedeutung, wenn alles Vertrauen, die Grundlage aller Geschäftsbeziehungen, immer auf Verwandtschaft oder verwandtschaftsartige rein persönliche Beziehungen gegründet blieb, wie dies in China sehr stark geschah. Die große Leistung der ethischen

\footnotetext{
${ }^{19}$ M. Hartmann, Die Komplexität des Vertrauens, in: M. Maring (Hg.), Vertrauen zwischen sozialem Kitt und der Senkung von Transaktionskosten, Karlsruhe 2010, 15-26, hier: 17f. Vgl. auch die Argumentation von K.E. Løgstrup, Die ethische Forderung, Tübingen 1959, 18: «Das Vertrauen steht nicht bei uns. Es ist gegeben. (...) In dieser Tatsache liegt die unausgesprochene, sozusagen anonyme Forderung an uns, das Leben des anderen, das das Vertrauen in unsere Hand legt, in unseren Schutz zu nehmen.»

${ }^{20}$ P. M. Blau, Exchange and Power in Social Life, New York/London 1964, 94.
} 
Religionen, vor allem der ethischen und asketischen Sekten des Protestantismus, war die Durchbrechung des Sippenbandes, die Konstituierung der Überlegenheit der Glaubens- und ethischen Lebensführungsgemeinschaft gegenüber der Wertegemeinschaft, in starkem Maße selbst gegenüber der Familie. Ökonomisch angesehen: die Begründung des geschäftlichen Vertrauens auf ethische Qualitäten der Einzelindividuen, welche in sachlicher Berufsarbeit bewährt waren. Die Folgen des universellen Misstrauens Aller gegen Alle, eine Konsequenz der offiziellen Alleinherrschaft der konventionellen Unaufrichtigkeit und der alleinigen Bedeutung der Wahrung des Gesichtes im Konfuzianismus, müssen ökonomisch vermutlich - denn hier gibt es keine Maßmethoden - ziemlich hoch veranschlagt werden. ${ }^{21}$

Sieht man von Webers eurozentrischen Vorurteilen einmal ab, so lässt sich seine Leitidee in den Gedanken fassen, dass die Globalisierung des Marktes von einem Ethos zehrt, dessen Ursprünge gleichermassen in der jüdisch-christlichen Religionsgeschichte wie der abendländischen Philosophie festzumachen sind.

Das philosophische Potential dieses Gedankens entfaltet Martin Hartmann in seiner Habilitationsschrift 〈Die Praxis des Vertrauens ${ }^{22}$ Im Anschluss an John Rawls vertritt Hartmann die These, dass ökonomische Vertrauenspraktiken nur dann langfristig stabil sind, wenn Vertrauen nicht rein instrumental - als Gleitmittel für eine expandierende Globalwirtschaft - gebraucht, sondern auch als intrinsisch wertvoll betrachtet wird. Anders gewendet: Bestimmte vertrauensvolle Einstellungen, deren ökonomische Bedeutung unbestritten ist, sind uns nur vor dem Hintergrund von gegebenen bzw. sich entwickelnden kulturellen, politischen und sozialen Praktiken möglich. Die für das wissenschaftliche Nachdenken über das Vertrauen interessante Frage ist nun, ob diese Praktiken selbst als vertrauensgenerierend oder lediglich als vertrauensbasierend $\mathrm{zu}$ beschreiben sind. Anders gefragt: Findet sich das Vertrauen in allen grundlegenden menschlichen Praxisformen oder gibt es solche, die auch ohne Vertrauen funktionieren und gerade als solche zur Basis von Vertrauensbeziehungen werden können?

Nicht zuletzt stellen sich in diesem Zusammenhang auch eine Reihe von gewichtigen empirische Fragen: Sind die vertrauentstabilisierenden Praktiken selbst hinreichend stabil, um die sich eigendynamisch entwickelnde globale Ökonomie aufzufan-

${ }^{21}$ M.Weber, Gesammelte Aufsätze zur Religionssoziologie. Bd. 1.Tübingen 1988, $523 \mathrm{f}$.

${ }^{22}$ Erscheint 2011 bei Suhrkamp. 
gen? Wie weit reichen die sozialen Vertrauensressourcen? Kann die Ökonomie selbst dazu beitragen, dass aus dem zerstrittenen Weltdorf eine "high-trust-society» wird? Während Skeptiker eine Erosion von Vertrauensressourcen konstatieren, ${ }^{23}$ verweisen optimistischere Prognostiker darauf hin, dass neue globale Vertrauensnetze am Wachsen sind. Das wohlverstandene Eigeninteresse der daran Beteiligten werde dafür sorgen, dass diese Netze dicht genug geknüpft werden. Dass die Möglichkeit, weltumspannende Vertrauensnetze aufspannen zu können, sehr verschieden genutzt werden kann, zeigt sich an einem der leistungsfähigsten globalen Vertrauensnetzwerke: Al Kaida.Vertrauen kann nicht nur missbraucht, sondern auch dazu benutzt werden, sich von anderen abzuschotten und zu mehr oder weniger aggressiven Gegengesellschaften zu formieren. Nicht nur unter Kindern, die sich gemeinsam verstecken, entsteht ein vertrauliches Zusammengehörigkeitsgefühl: Auch die Krieger, die sich gemeinsam im trojanischen Pferd oder einem feindlichen Flugzeug verschanzen, dürften etwas Ähnliches erleben. Doch gibt es nicht auch eine Form vonVertrauen, die durch ihren universalistischen Zug solche Mechanismen unterläuft? Ein Grund- oder Metavertrauen, das sich einer exklusiven Vertrauensvergabe widersetzt?

\section{Grundvertrauen}

An dieser Stelle stossen die bisherige empirische Vertrauensforschung und die gegenwärtigen Vertrauensdiskurse auf ein noch weiter zu bearbeitendes Problemfeld. Zum einen konzeptionell: Lässt sich ‘Grundvertrauen` (basic trust) überhaupt in kohärenter Weise begrifflich fassen und den anderen Formen von Vertrauen zuordnen? Zum anderen ist Grundvertrauen empirisch schwer fassbar. In der klinisch-psychologischen Forschung, von der man diesbezüglich Klärung erhoffen könnte, sucht man vergeblich nach empirischen Studien. Schliesslich gibt es auch eine gewisse Nähe zwischen Grundvertrauen und religiösem Vertrauen, die Fragen aufwirft: Bedarf das Grundvertrauen, will es nicht ins Leere gehen, einer

\footnotetext{
${ }^{23}$ Vgl. D.Z. Phillips, On Trusting Intellectuals on Trust, in: Philosophical Investigations 25 (2002), 33-53, hier 51: «[I]f a dominant morality in a society is one which places a high regard on security, planning in the event of contingencies, this will erode conceptions of absolute trust. It is easy to see how marriage vows could be affected (and have been). How can one give absolute trust to another when the future is so uncertain? (...) Unconditional vows would be deemed irrational, whereas conditional vows would seem like common sense.»
} 
Fundierung in einem letzten Grund? Lebt im Grundvertrauen einer säkularen Gesellschaft ein Glauben fort, der von vielen als überholt angesehen wird? Religionssoziologische Studien belegen zumindest, dass das Vertrauen in eine umfassende Ordnung, ohne die zu leben schwierig ist, bis in die Gegenwart hinein religiös gefärbt ist. Ähnlich wie es das Vertrauen in die Sicherheit derVerkehrsmittel, die man benutzt, nachweisbar stärkt, wenn man den Expertensystemen, die sie betreiben, vertrauen kann, so stärkt es offensichtlich das Vertrauen in die Güte und Zuverlässigkeit der Weltordnung, wenn man ihrem Ursprung bzw. ihrem Urheber vertrauen kann. Wie verändert sich aber das Lebens- und Weltvertrauen, wenn das religiöse Grundvertrauen, dass dieses zu rechtfertigen vermag, in Frage gestellt oder zumindest nicht ausdrücklich kultiviert wird?

Aus entwicklungspsychologischer Sicht liesse sich argumentieren, dass religiöses Grundvertrauen lediglich eine unter vielen möglichen Transformationsformen eines frühkindlichen «Urvertrauens〉 darstellt. Was sind die Alternativen? Kann ein säkulares Welt- und Lebensvertrauen eine ähnliche Funktion erfüllen? Auch ein säkulares Grundvertrauen bedarf jedenfalls einer gemeinschaftlichen Abstützung. Doch hängt eine solche ohne weitere - religiöse Rückbindung nicht in der Luft? Man kann aber auch umgekehrt fragen: Ist nicht gerade die Suche nach letzten Sicherheiten, nach einem festen Fundament, auf das man sich in Leben und Tod verlassen kann, Ausdruck fehlenden Vertrauens? Gehört es nicht zu den Errungenschaften der Moderne, gelernt zu haben, dass man dem Leben auch ohne letzte Gründe vertrauen kann? Dass man sich auch ohne religiöse Gewissheiten auf die Stabilität natürlicher Rhythmen und kultureller Ordnungen verlassen kann? Lässt es sich nicht ganz gut in einer Welt leben, die kein ewiges Firmament und keine göttliche Vorsehung mehr erkennen lässt? Kann nicht gerade, wie Richard Rorty zu Bedenken gegeben hat, aus der geteilten Erfahrung einer letzten Ungesichertheit Solidarität und Mitmenschlichkeit entstehen?

$\mathrm{Ob}$ es angemessen ist, religiöses Vertrauen oder christliches Gottesvertrauen als Grundvertrauen zu konzeptionalisieren bzw. dieses mit jenem direkt zu verknüpfen, darüber wird auch theologisch gestritten. Einig ist man sich vermutlich allein darin, es als eine Manifestationsform des Unglaubens zu betrachten, wenn kirchliche Gemeinschaften auf die Verunsicherungen der (Post-)Moderne mit religiöser Selbstsicherung reagieren. Wie sind aber Gottesvertrauen und Grundvertrauen miteinander verknüpft? Im Anschluss an Karl 
Rahner ${ }^{24}$ kann man versuchen, die Erfahrung, dass basales Vertrauen sich in einer instabilen Welt oft auch ohne religiöse Letztversicherung zu bewähren scheint, selbst theologisch zu deuten und zu unterfangen: als Folge der vertrauensstiftenden Präsenz des Geistes, der in den Menschen den Sinn für den Sinn des Ganzen wachhält. Doch ist das nicht eine theologisch überzogene Deutung weltlicher Phänomene? Ist «säkulares Grundvertrauen〉 nicht ein etwas allzu feierliches Wort für eingespielte Gewohnheiten und kumulierte Erfahrungen von Verlässlichkeit?

In historischer Perspektive fragt sich, ob die Genese der modernen Zivilgesellschaft nicht als ein komplexer Transformationsprozess $\mathrm{zu}$ verstehen ist, in dem sich an die Stelle des nicht mehr allgemein vorauszusetzenden Gottesvertrauens kulturelle Ersatzformen herausbilden. Während Ute Frevert einer solchen Sicht zugeneigt scheint, ${ }^{25}$ steht Martin Hartmann der Möglichkeit, eine solche Fortschrittsgeschichte des Vertrauens schreiben zu können, skeptisch gegenüber. In seiner bereits genannten Habilitationsschrift vertritt er die moderatere These, dass sich über die verschiedenen Formen und Praktiken gesellschaftsprägenden Vertrauens, die in einem komplexen genealogischen Verhältnis zueinander stehen, die Relevanz des intrinsischen Moments durchhält. So partizipieren auch die Bürgerinnen und Bürger moderner Demokratien, ohne dass sie dies ausdrücklich wahrnehmen, an einer auf gegenseitiger Anerkennung basierenden Vertrauenskultur, die ihnen einen verlässlichen Lebensrahmen gibt. Als Nutzniesser dieser Kultur des Vertrauens, so Hartmanns moralphilosophische Schlussfolgerung, tragen sie auch Verantwortung für sie. "Einzig in dem Maße, in dem wir uns als Glieder einer kollektiven Anerkennungsordnung verstehen, können wir das gleichsam sozialisierte Wohlwollen als Basis für ein Vertrauen unter sonst Fremden betrachten. ${ }^{26}$

Das gesellschaftlich vermittelte und akkumulierte Vertrauen, von dem das Grundvertrauen des Einzelnen in ähnlicher Weise lebt wie vormals vom kirchlich vermittelten religiösen Vertrauen, ist insofern gefährdet, als der intrinische Wert des Vertrauens im Kontext der instrumentellen Vertrauenspraxis der Ökonomie leicht vergessen wird. Wo Vertrauen nur als hilfreiches Mittel zu einem bestimmten Ziel betrachtet wird, etwa zur Senkung von Transaktionskosten oder

\footnotetext{
${ }^{24}$ K. Rahner, Bietet die Kirche letzte Gewishheiten?, in: ders., Schriften zur Theologie, Bd. 10, Zürich/Einsiedeln/Köln 1972, 286-304.

${ }^{25}$ U. Frevert, Vertrauen - eine historische Spurensuche, in: dies., (Hg.), Vertrauen. Historische Annäherungen, Göttingen 2003, 7-66.

${ }^{26}$ M. Hartmann, Die Komplexität des Vertrauens (s. Anm. 19), 24
} 
zur Herstellung eines guten Arbeits- oder Verkaufsklimas, wird die demokratische Vertrauenspraxis, die die Wohlfahrt der einzelnen und der Gemeinschaft institutionell absichert, destabilisiert. ${ }^{27}$ Der intrinsische Wert sozialen Vertrauens liegt nach Hartmann schlicht darin, dass es nicht nur zu unserer persönlichen, sondern auch zu unserer politischen Lebensform gehört, einander vertrauen zu können. Im Anschluss an John Locke und Shmuel Eisenstadt plädiert Hartmann deshalb für ein ethisches Vertrauensmodell, das vom Gedanken einer kooperativen Autonomie ausgeht. Unser Vertrauen verdienen besonders diejenigen, die es in der Beziehung zu uns nicht nur instrumentell gebrauchen, sondern es als Wert an sich schätzen: «Wir müssen im Vertrauen davon ausgehen, dass unser Kooperationspartner ein Interesse am Vertrauensverhältnis als solchem hat, das über sein Eigeninteresse hinaus geht. ${ }^{28} \mathrm{Um}$ solche Vertrauensverhältnisse zu ermöglichen und zu stabilisieren, bedarf es nach Hartmann bewährter Praktiken, in denen sich die intrinsische Güte des Vertrauens wahrnehmbar darstellen.

\section{Metavertrauen}

Wenn die Vertrauensdiskurse der letzten Jahre etwas bewirkt haben, dann gewiss eine Sensibilisierung für das Phänomen des Metavertrauens. Nach Niklas Luhmann ermöglicht ein Vertrauen in die Macht desVertrauens Handlungen, die durch einfaches Vertrauen allein nicht zustande kommen können. ${ }^{29}$ Luhmann neigt dazu, diese reflexive Aufstufung des Vertrauens - und nicht lediglich das Reflexivwerden (der Tragweite) des Vertrauens - als Errungenschaft der Moderne zu betrachten. Es fragt sich allerdings, ob es sich nicht eher um eine Neubeschreibung eines Phänomenfelds handelt, das sich im Zuge der Modernisierung erweitert hat, aber auch vorher schon bedeutsam gewesen sein dürfte. Auch das Selbstvertrauen und ebenso gewisse Formen des Fremdvertrauens lassen sich nämlich, genauer betrachtet, als Metavertrauen beschreiben. Beruht Selbstvertrauen doch nicht zuletzt in einem Vertrauen auf das Vertrauen, das andere mir schenken, während unser Vertrauen in

${ }^{27}$ Vgl. M. Hollis, Trust within reason, Cambridge/New York 1998, 159.

${ }^{28} \mathrm{M}$. Hartmann, Wer hat unserVertrauen verdient? Philosophische Kriterien derVertrauenswürdigkeit, in: M. Fischer/I. Kaplow (Hg.), Vertrauen im Ungewissen. Leben in offenen Horizonten, Münster 2008, 48-69, hier 67.

${ }^{29}$ N. Luhmann, Vertrauen. Ein Mechanismus der Reduktion sozialer Komplexität. Stuttgart ${ }^{4} 2000,91$. 
eine bestimmte Person oft vom Vertrauen geweckt wird, das andere ihr schenken, und es insofern als ein Vertrauen in das Vertrauen der anderen beschreiben lässt.

Von Metavertrauen kann in diesen Fällen allerdings nur in einem schwachen Sinne gesprochen werden. Lässt sich doch bezweifeln, ob die vorgeschlagene Beschreibung des Selbst- und Fremdvertrauens als «Vertrauen in das Vertrauen der anderen〉 angemessen ist:Vertrauen wir doch den betreffenden Personen als solchen und nicht einem von ihnen isolierbaren Vertrauen. Eine eindeutigere Form von Metavertrauen findet sich vielleicht dort, wo sich jemand ausdrücklich dafür entscheidet, in einem bestimmtem Bereich - oder generell - vertrauensvollem Handeln den Vorzug zu geben. Wenn etwa ein Managementtraining das Ziel verfolgt, die Wirksamkeit von Vertrauen aufzuzeigen und angemessene Formen von innerbetrieblicher Vertrauensvergabe bzw. der Aufbau eines Vertrauensklimas eingeübt werden, dann wird Metavertrauen ausgebildet und aufgebaut. Aufgrund der vermittelten Erfahrungen beginnen die Teilnehmer eines solchen Trainings auf das (bzw. auf eine bestimmte Form von) Vertrauen zu vertrauen. Ein anderes Beispiel bildet die bei jungen mexikanischen Einwandern in den USA beobachtete Praxis der "confianza en confianza», eines vertrauensgenerierenden Networking, das die sozialen Chancen einer Minorität besser zu wahren vermag als das sonst bei Jugendlichen übliche kompetitive Verhalten. ${ }^{30}$

Bei allen bisher genannten Formen des /Vertrauens in Vertrauen〉 handelt es sich strenggenommen nicht um ein Reflexivwerden eines bestimmten Vertrauens, sondern um jeweils zwei wohl zu unterscheidende Formen von Vertrauen. Wir vertrauen nicht mit demselben Vertrauen an die Wirksamkeit des interpersonalen Vertrauens, mit dem wir anderen Menschen vertrauen. In den meisten Fällen nähert sich ein solches Metavertrauen vielmehr einem nicht mehr personal gefärbten Sich-Verlassen (reliance) an. Wir verlassen uns - als Manager, Lehrerinnen oder Seelsorger - in bewussten Formen des Kontrollverzichts und der Kompetenzübertragung auf die transformierende und kreative Kraft des Vertrauens. Und Analoges liesse sich sagen über das (Meta-)Vertrauen in die Leistungskraft des Misstrauens.

Gibt es nicht noch anspruchsvollere Formen des Metavertrauens? Ist es nicht auch möglich, demVertrauen so vertrauen, wie man die Liebe lieben kann? Und käme ein solches Vertrauen dem Gottesvertrauen

\footnotetext{
${ }^{30}$ Vgl. I. R. Martin, Insights into the Complexities in Persisting Latina College Students, Univ. of Massachusetts/Amherst 2010, 28f., abrufbar unter (15.09.2010): http://scholarworks.umass.edu/cgi/viewcontent.cgi? article $=1173 \&$ context $=$ open access dissertations
} 
nahe? Theologisch fragt sich jedenfalls, ob Gottesvertrauen nicht eher als Metavertrauen denn als Grundvertrauen zu beschreiben wäre: als Vertrauen in Gottes Vertrauen. Auch hier könnte man einwenden, dass sich das Gottesvertrauen zunächst und vor allem auf Gott selbst richtet und nicht auf sein Vertrauen. Wenn aber in Gott Sein und Wirken ineins fallen, dann könnte Metavertrauen als neue theologische Kategorie in den theologischen Diskurs eingeführt werden: Um ausdrücklich zu machen, dass in christlicher Perspektive religiöses Vertrauen nicht in erster Linie als Vertrauen in eine ewige Weltordnung zu beschreiben ist, sondern eben als Vertrauen in die Verlässlichkeit des göttlichen Vertrauens. ${ }^{31}$

${ }^{31}$ Man kann an dieser Stelle theologisch weiterfragen, ob das Vertrauen Gottes als trinitarisch differenziertes Selbstvertrauen zu beschreiben ist oder eher als Fremdvertrauen in Gestalt eines Vertrauen, dass der nicht eben vertrauenswürdige Mensch schliesslich das in ihn gesetzte Vertrauen zu rechtfertigen vermag. 